\title{
The landscape of congenital heart disease
}

\author{
Giovanni Biglino \\ Bristol Heart Institute, School of Clinical Sciences, University of Bristol, Bristol, UK \\ Correspondence to: Giovanni Biglino. Bristol Heart Institute, School of Clinical Sciences, University of Bristol, Bristol, UK. Email: g.biglino@bristol.ac.uk.
}

Submitted Nov 22, 2016. Accepted for publication Jan 19, 2017.

doi: $10.21037 /$ cdt.2017.03.08

View this article at: http://dx.doi.org/10.21037/cdt.2017.03.08

The soft echo of visitors' voices in the tall space, the noise of old wooden choir benches, the intriguing display of white coral-like structures under bell jars, structures that turn out to be hearts with a range of repaired congenital defects. It's just a pain is a multimedia site-specific installation, created by artist Sofie Layton within the context of the Feel It Festival. The festival is an interdisciplinary exploration of pain and breathlessness and the setting is the striking space of St Paul's Church in the Georgian Portland Square in Bristol. As part of the event, Layton's installation in the choir of the church (Figure 1) is a representation of the landscape of congenital heart disease, merging the rigour of medical language and anatomical forms with the story of boy receiving heart transplantation told by his mother in a moving soundscape that visitors can listen to on headphones, sitting in the pews of the choir. It is a delicate piece, in the way in which voices and stories of illness are handled, but powerful in both setting and content.

There are several layers to the work. Firstly, a series of six textile panels visually represents key components of the journey of a patient with congenital heart disease, from cardiovascular anatomy to the name of the defects themselves, from medications to technological innovations that facilitate treatment, as well as the narrative component. The latter is conveyed through a series of statements that have emerged during conversations with patients and families at the time of the artist's residency at Great Ormond Street Hospital for Children in London (2015-2016), short sentences that bring in the human component and make it central to the visual representation. The linguistic element is intertwined on the panels with the anatomical element, i.e., heart outlines (recreated from magnetic resonance imaging, MRI, data) brought to life in metallic shades that reflect the light filtering through the church's stained windows (Figure 2).

Secondly, a metal table, echoing the surgical theatre but somehow also echoing the altar just there in the back, is placed in between the choir's pews, and a display of heart models is mounted on it. The white forms, so organic and alive, have been generated by means of 3D printing technology starting from MRI data. The artist has placed the models (which present a series of congenital conditions including repaired tetralogy of Fallot, palliated hypoplastic left heart syndrome or a case of total anomalous pulmonary venous drainage) under bell jars, transforming the medical models into museum objects, a visual manifestation of the complexity and the beauty of cardiovascular anatomy (Figure 3).

Thirdly, the whole installation comes to life and breaths the patient's story (the unknown patient, yet so central, so present in the piece). A soundscape, realized purposely by the artist in collaboration with composer Jules Maxwell, can be listened to while sitting in the choir benches, and the use of headphones allows to really sink into the piece, in a space (the church) that in itself commands silence and encourages contemplation (Figure 4). The soundscape includes a hint to church music, evoking the setting, but mixed with medical sounds, particularly the sound of the MRI scanner. The narrative element of the landscape now becomes explicit, with a mother recounting her child's heart transplantation. The medical language element also is recurrent, in a succession of words that are read from the textile panels by the artist herself. Technical language, somewhat aseptic, but integral to the narration. And language that reverberates in the forms of heart outlines and heart models.

By creating the installation in the church setting, the artist also touched on the use of the heart in ecclesiastic imagery, and votives in particular (ex voto, or milagros), where the language becomes looser, more poetic, a prayer, an offering; where a leg may represent a pilgrimage, and a heart may represent a romance.

The piece as a whole brilliantly captures the complexity 


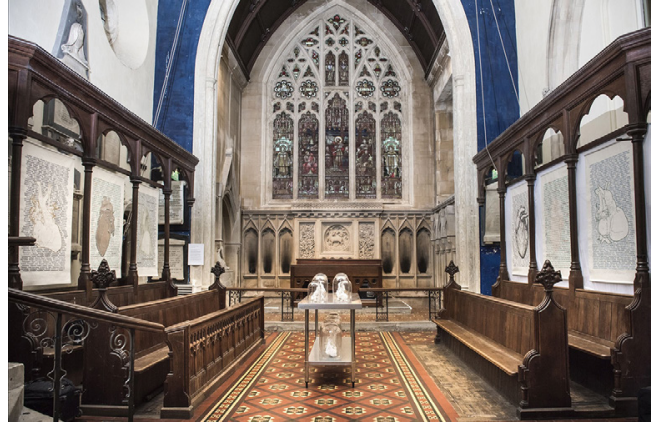

Figure 1 The choir of St Paul's Church (Bristol) where It's just a pain was installed by artist Sofie Layton.

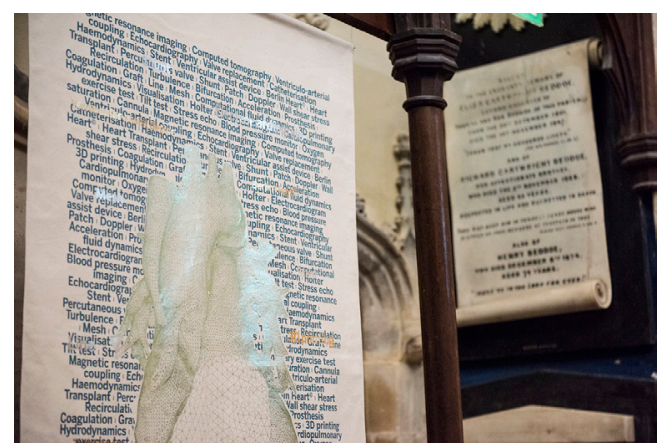

Figure 2 Detail of one of six panels that explore the language of key themes in the journey of a patient with congenital heart disease, in this case technologies and devices that aid diagnosis and treatment.

of the world of congenital heart disease, its language, its forms, its life-long nature. It is an aspect of the latter that inspired the title of the installation. During a conversation in the hospital, the mother of a teenage patient recounted that she would sometimes see her son touching his side or his chest, and well knowing the implications she would enquire: "Are you okay?", to which he would simply answer: "It's nothing, it's just a pain".

\section{Acknowledgements}

It's just pain was explored and researched by Sofie Layton at Great Ormond Street Hospital for Children in London during her residency (2015-2016) which culminated in the exhibition titled Under the Microscope. The soundscape is a collaboration between Sofie Layton and composer Jules Maxwell. The 3D heart models were created by Dr. Giovanni Biglino and the Clinical Cardiovascular Engineering team at University College London/Great

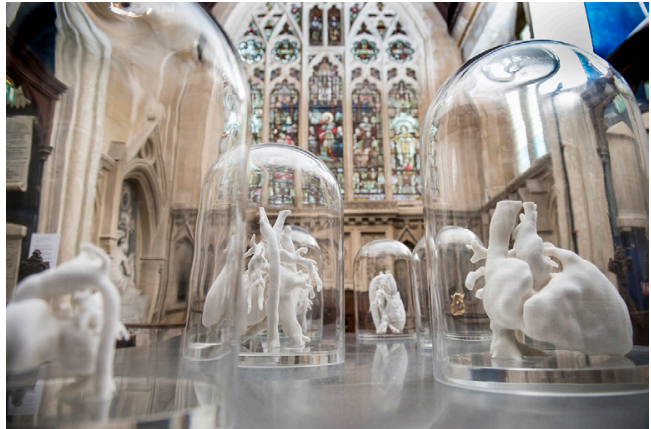

Figure 3 Models of hearts with congenital defects manufactured by means of 3D printing technology are exhibited under bell jars.

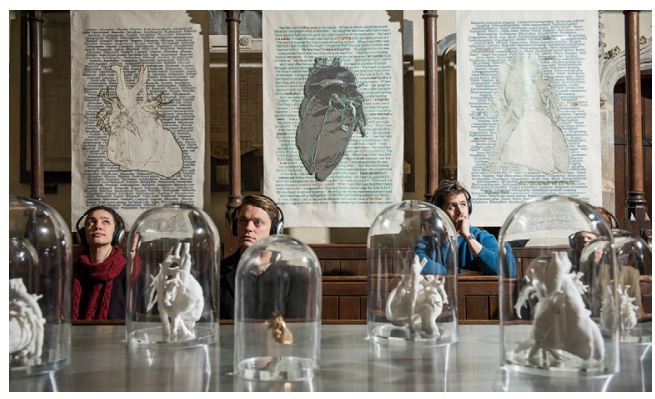

Figure 4 Visitors listen to the sound component of the piece while immersed in the landscape of congenital heart disease.

Ormond Street Hospital. The Feel It Festival, curated and produced by Georgia Bladon, took place on 17-20 November 2016 in Bristol and was an endeavour supported by the University of Bristol's Elizabeth Blackwell Institute for Health Research and the Life of Breath Project, in collaboration with Circomedia, made possible by the generous support of the Wellcome Trust. The installation was also specifically supported by the Bristol Heart Institute. All images (C) Stephen King. Artist Sofie Layton approved this article.

\section{Footnote}

Conflicts of Interest: The author has no conflicts of interest to declare.

Cite this article as: Biglino G. The landscape of congenital heart disease. Cardiovasc Diagn Ther 2017;7(Suppl 1):S55-S56. doi: $10.21037 / \mathrm{cdt} .2017 .03 .08$ 